\title{
Neuromyelitis optica spectrum disorders with unevenly clustered attack occurrence
}

Tetsuya Akaishi, MD, PhD, Ichiro Nakashima, MD, PhD, Toshiyuki Takahashi, MD, PhD, Michiaki Abe, MD, PhD, Tadashi Ishii, MD, PhD, and Masashi Aoki, MD, PhD

Neurol Neuroimmunol Neuroinflamm 2020;7:e640. doi:10.1212/NXI.0000000000000640

\section{Abstract}

\section{Objective}

The aim of this study was to elucidate the characteristics of clinical attacks in neuromyelitis optica spectrum disorders (NMOSDs) with positive serum anti-aquaporin-4 antibody. Both the timing and sequential pattern of clinical types were analyzed.

\section{Methods}

A total of 69 patients with NMOSD were enrolled in this study, all of whom were treated at a single university hospital. All data regarding the clinical attacks (including types and date) together with other clinical information were collected.

\section{Results}

Analysis of clinical attacks from the enrolled patients showed that there were 2 distributional patterns of attack occurrence in each patient: (1) "clustered" occurrences, which occurred within 12 months from the previous attack, and (2) "nonclustered" intermittent occurrences, which occurred $\geq 12$ months after the previous attack. These occurrences were regardless of the duration from the onset. During the "clustered" period, clinical attacks were more likely to show a similar clinical manifestation, such as optic neuritis or myelitis. After entering the "nonclustered" intermittent period, the relapses were of random clinical type, regardless of the previous clinical manifestation.

\section{Conclusions}

Patients with NMOSD showed mixed periods of "clustered" occurrence with frequent attacks presenting with similar manifestations and "nonclustered" intermittent periods with sparse relapses. Approximately half of the relapses occurred during the "clustered" period within 12 months of the last clinical attack. Clinicians should pay special attention to whether the patients are presently in the "clustered" or "nonclustered" period to decide optimal relapse-preventive strategies.

\author{
Correspondence \\ Dr. Akaishi \\ t-akaishi@med.tohoku.ac.jp
}




\section{Glossary}

AQP4 = aquaporin-4; ARR = annualized relapse rate; NMOSD = neuromyelitis optica spectrum disorder; $\mathbf{O N}=$ optic neuritis.

Neuromyelitis optica spectrum disorder (NMOSD) is an autoimmune disorder characterized by the presence of serum anti-aquaporin-4 (AQP4) autoantibody, recurrent optic neuritis (ON), and myelitis in most cases. ${ }^{1,2}$ Unlike MS, neurologic disability in patients with NMOSD typically accumulates with each clinical episode. ${ }^{3,4}$ Thus, suppressing relapses is crucial in the management of NMOSD, and elucidating the characteristics and pathomechanisms of the relapses is essential. ${ }^{5}$ However, at present, little is known about the relapse timing and sequential patterns of clinical attacks in patients with NMOSD. As a result, it is challenging to evaluate the patients' level of clinical severity and disease activity in clinical trials or when considering therapeutic options.

In this study, we aimed to elucidate the chronological characteristics of clinical relapses in NMOSD by applying time series analyses for each patient. We also evaluated the possible association between relapse timing and the sequential pattern of clinical attacks.

\section{Methods}

\section{Patient enrollment and variables evaluated}

A total of 69 consecutive patients with serum anti-AQP4 antibody-positive NMOSD, who were treated in our university hospital and followed up for more than 10 years until June 2019, were enrolled. All patients were confirmed to be serum positive for the anti-AQP4 antibody using a cell-based assay, which is described below.

The following demographic and clinical variables were collected from the 69 enrolled patients: sex, onset age, disease duration, data pertaining to the clinical attacks (types and date of each attack), and timing of the implementation of the relapse-preventive therapy.

\section{Serum anti-AQP4 antibody testing}

We conducted a microscopic live cell-based assay for AQP4 based on previous reports. ${ }^{6-8}$ In brief, human M23-AQP4expressing HEK293 cells were incubated with 1:16 diluted serum samples and then stained with Alexa 488-conjugated secondary antibody. The positivity of the antibody was detected based on fluorescence emission using fluorescence microscopy.

\section{Evaluated outcomes}

The 2 major outcomes evaluated in this study were (1) the sequential pattern of the types of manifested clinical attacks (i.e., ON, myelitis, medullary, and cerebral) and (2) time series analysis of relapse timings in each patient. After we confirmed the presence of irregularity in the relapse timing, we additionally investigated the possible effect of uneven relapse timing on the observed sequential pattern of clinical symptoms.

For the analysis of the sequential pattern of clinical attacks, data from 50 of the 69 enrolled patients, who experienced clinical relapse at least once by June 2019, were used. Clinical attacks with $\mathrm{ON}$ and myelitis were counted, but those with medullary lesions (i.e., hiccups/nausea/vomiting) or cerebral lesions were excluded from the sequential analyses of clinical manifestations for accurate interpretation; the details of the excluded data and effect of excluding them on the obtained results are described in subsequent sections.

For the analysis of the irregularity of relapse timing, data from both the 50 patients with relapses and 19 patients without relapses were used. Data on the elapsed time period (months) from the last clinical attack were collected from the 50 patients with relapses, whereas those on the relapse-free period (months) from the last attack to June 2019 were collected from all 69 enrolled patients. Moreover, these data were separately evaluated based on the administration of oral relapse-preventive therapies. All 69 enrolled patients were eventually treated with low-dose oral corticosteroid, with or without other maintenance treatments (11 patients with azathioprine, 2 with mycophenolate mofetil, and 1 with tacrolimus). Data of relapse timing during medication-free periods were collected from 36 patients who were not administered relapse-preventive therapies from the onset, whereas data during treatment with oral corticosteroid were collected from all 69 enrolled patients.

\section{Relapse frequency with no medication by the time from clinical onset}

First, we estimated the mean annualized relapse rate (ARR), which was calculated as the total number of relapses divided by the total person-time of follow-up, in the following 2 time periods: $0-24$ months from clinical onset (36 patients) and the following $24-48$ months from onset (21 patients).

The relapse-free period without relapse-preventive therapies was then compared between the first 24 months from clinical onset (36 patients) and the next 24 months in patients without relapses in the first 24 months (14 patients). The purpose of this analysis was to compare the probability of experiencing relapses in the near future between patients who have a history of relapses during the previous 24 months and patients without relapses during the previous 24 months.

\section{Statistical analysis}

For the comparisons of frequency using $2 \times 2$ tables, a $\chi^{2}$ test or Fisher exact test was implemented based on the sample size of each cell. Comparisons of the frequency with $\mathrm{k} \times 2$ tables $(\mathrm{k}>2)$ 
were performed using a Cochran-Armitage trend test. The elapsed time periods from onset and from the last clinical attack for each clinical attack during the medication-free period were compared using the Mann-Whitney $U$ test because both were nonnormally distributed. The relapse-free period without medication between 0-24 months and 24-48 months from onset was compared using Kaplan-Meier survival analysis. Statistical analyses were conducted using either SPSS Statistics Base 22 software (IBM, Armonk, NY) or MATLAB R2015a (MathWorks, Natick, MA).

\section{Institutional Review Board}

This study was approved by the Institutional Review Board of Tohoku University Hospital (IRB No. 2010589). Written informed consent was obtained from all enrolled patients.

\section{Data availability}

Qualified researchers may obtain deidentified clinical data used in the current study from the corresponding author on reasonable requests, such as revalidation of the procedures and results.

\section{Result}

\section{Clinical information from the enrolled patients}

Of the 69 enrolled patients, 66 (95.7\%) were women and the remaining 3 (4.3\%) were men. The mean and SD of the onset age was $42.7 \pm 14.1$ years. The months in which all clinical episodes occurred (i.e., 342 attacks in total) showed no uneven seasonal distribution. The mean \pm SD disease duration in 2019 was $19.7 \pm 10.7$ years. Thirty-six of the 69 patients $(52.2 \%)$ were not given long-term relapse-preventive treatments after their first clinical attack (onset). As for the clinical manifestation of the 342 clinical attacks, 110 were ON (32.2\%), 203 were myelitis (59.4\%), 19 were medullary lesions (5.6\%), and 10 were cerebral lesions (2.9\%). The rate of myelitis based on age at the time of the attack was $46.3 \%$ for $11-30$-year-olds, $52.3 \%$ for $31-40$-year-olds, $67.0 \%$ for $41-50$-year-olds, and $62.9 \%$ for 51-80-year-olds, showing an increased frequency of myelitis with age ( $p=0.0186$, Cochran-Armitage trend test).

\section{Histogram of the ratio of optic neuritis episodes in the enrolled patients}

If the enrolled patients show a bimodal distribution for the ratio of ON episodes, such a distribution could affect the following analyses of the sequential pattern and unevenness of relapse timings. To exclude this possibility, we present a histogram of the $\mathrm{ON}$ ratio calculated in each of the 43 patients with 3 or more clinical attacks, including onset (figure 1). As shown in figure 1, the distribution of the $\mathrm{ON}$ ratios showed a monotonic decrease without bimodality. In other words, there was no peak of patients who exclusively presented with ON episodes without myelitis episodes.

\section{Time series analysis for the timing of clinical attacks}

Next, we evaluated the timing and density of clinical attacks. The occurrence timing and clinical types of each attack in the
Figure 1 The number of patients with $\geq 2$ attacks based on the rate of attacks with optic neuritis (ON)

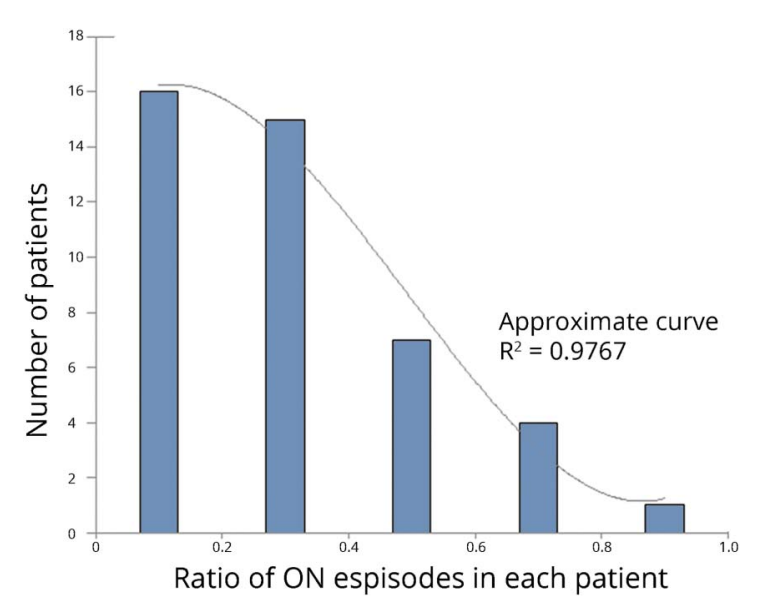

$\mathrm{ON}=$ optic neuritis

43 patients with 3 or more clinical attacks are presented in figure 2. The presented clinical course in each patient suggests an unevenness in the density of relapse occurrence, irrespective of the duration from onset.

Based on this result, we evaluated the elapsed time from the last clinical attack (figure 3A) for each clinical episode of the enrolled patients. The relapse-free period from the last clinical attack in June 2019 is also shown in the histogram. As shown in these figures, clinical relapses in NMOSD were significantly likely to densely cluster with a time period between attacks of $<12$ months.

To exclude the possibility of the effect of treatments, we also depicted the histograms of the time period between 2 tandem episodes based on the presence of oral relapse-preventive therapy (i.e., low-dose oral corticosteroid in this study). Both the time period between attacks without treatment (figure 3B) and with relapse-preventive treatment (figure 3C) reproduced a significantly skewed deviation with a peak at an intervening time period of $<12$ months. These histograms suggest that approximately half of the relapses in patients with NMOSD take place in the "clustered" period within 12 months of the last attack, irrespective of the administration of relapse-preventive therapy.

\section{Relapse frequency with no medication based on the time from onset}

Next, the likeliness of experiencing relapses based on the time from the last clinical attack (i.e., 0-24 months vs 24-48 months) among patients without relapse-preventive therapy after the onset $(n=36)$ was evaluated. The estimated mean ARR in the "0-24 months from onset" period was 0.47 (95\% CI: $0.35-0.60 ; \mathrm{n}=36$ ) and that in the " $24-48$ months from onset" period was 0.25 (95\% CI: $0.12-0.38 ; n=21$ ), which was significantly higher in the former group $(p=0.0259)$. Kaplan-Meier survival analysis to compare the relapse-free 


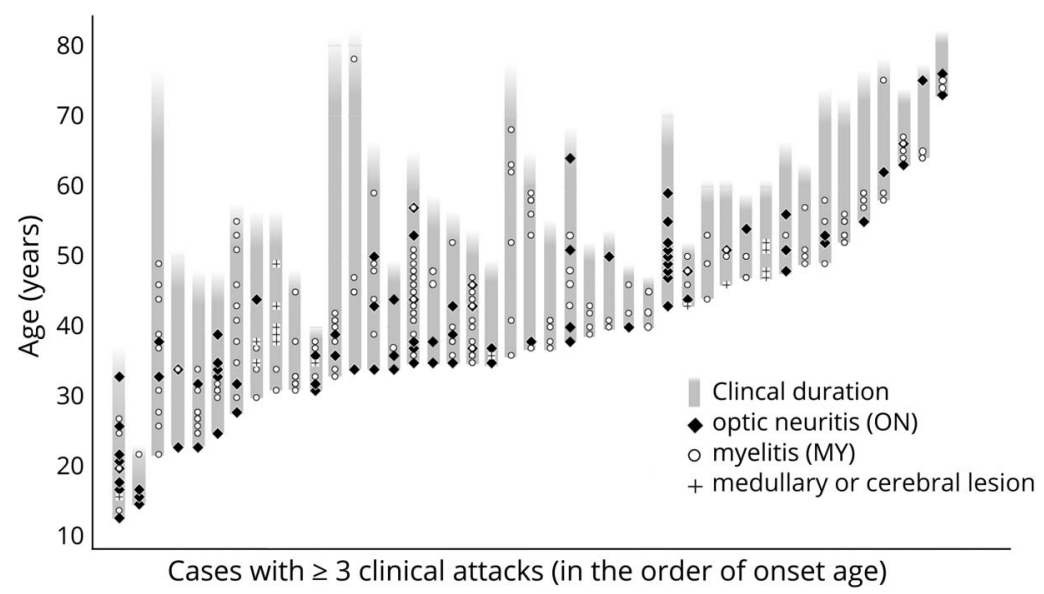

The presented clinical courses imply a clustered occurrence of attacks, irrespective of the duration from clinical onset. period between the 2 time periods was performed. For this analysis, data of the "0-24 months from onset" period was obtained from all 36 patients and data of the "24-48 months from onset" period was obtained from the 14 patients who did not experience a relapse during the first 24 months after the onset. The obtained Kaplan-Meier curves (figure 4) revealed that the "0-24 months from onset" period showed a significantly shorter relapse-free period from onset with a lower achievement rate of a relapse-free condition by the end of the follow-up period, compared with the "24-48 months from onset” period ( $p=0.0206$, generalized Wilcoxon test).

\section{Sequential pattern of attack types by the time from the last attack}

Next, we studied the sequential pattern of attacks in each patient. The sequential pattern of clinical relapse in the 50 patients with relapses is summarized in the upper half of table. For all 3 types of analyses by the number of sequential attacks, the latest preceding attack significantly affected the following clinical relapse ( $p=0.0058$ for 2 tandem episodes, $p=0.0015$ for 3 tandem episodes, and $p<0.0001$ for 4 tandem episodes).

Because patients with NMOSD showed a clustered pattern of attack, irrespective of the duration from onset, we added time series analyses for the 2 sequential clinical episodes after dividing the episodes into those during the "clustered" period and those during the "nonclustered" intermittent period. These data from the sequentially repeated 2 clinical relapses for each time period between attacks are summarized in the lower half of table. As presented, the last attack type was likely to be repeated during the "clustered" period ( $p=0.0203$ for analysis within a 6-month period, and $p=0.0006$ for analysis within a 12-month period). However, the following type of clinical episode occurred completely randomly and was not affected by the last attack after the "nonclustered" intermittent period ( $p=0.78$ for analysis after a 12 -month period, and $p=$ 1.00 for analysis after a 24-month period).
For reference, episodes of $\geq 2$ attacks with medullary lesions were seen in 2 patients (one patient with 5 attacks and another with 2 attacks), one of whom experienced tandemly repeated attacks with medullary lesions 5 times. Episodes of $\geq 2$ attacks with cerebral lesions were seen in 1 patient, who exclusively experienced attacks only with cerebral lesions 4 times during her entire clinical course. The abovementioned conclusions about the sequential pattern of attack types in NMOSD did not change even when we provisionally incorporated the episodes with brain lesions in the sequential analyses after supposing them as attacks of ON or myelitis.

\section{Discussion}

In this report, we demonstrated that patients with NMOSD showed a mixed clinical course of "clustered" periods (repeated relapses at $<12$ months from the last attack) and "nonclustered" intermittent periods (repeated relapses at $\geq 12$ months from the last attack). Time series analysis of the relapse type in each patient showed that a similar clinical type of episode was likely to be repeated during the "clustered" period, but not after entering the "nonclustered" intermittent period. In other words, if a patient with NMOSD does not experience relapses for more than a specific period of time, the patient can be regarded as being in a "nonclustered" intermittent period, and the timing or clinical type of the following relapse cannot be predicted by the preceding clinical attacks.

The results of this study suggest that clinicians should pay specific attention to whether their patients are presently in the "clustered" period, with a high likelihood of relapse frequency, or in the "nonclustered" intermittent period, with a low likelihood of relapse frequency, because this could affect the appropriate type of relapse-preventive therapy in each patient at different time points. This likelihood of relapse appears to be independent of the duration from the onset or the administration of oral immune suppressants (i.e., low-dose oral corticosteroid in this 


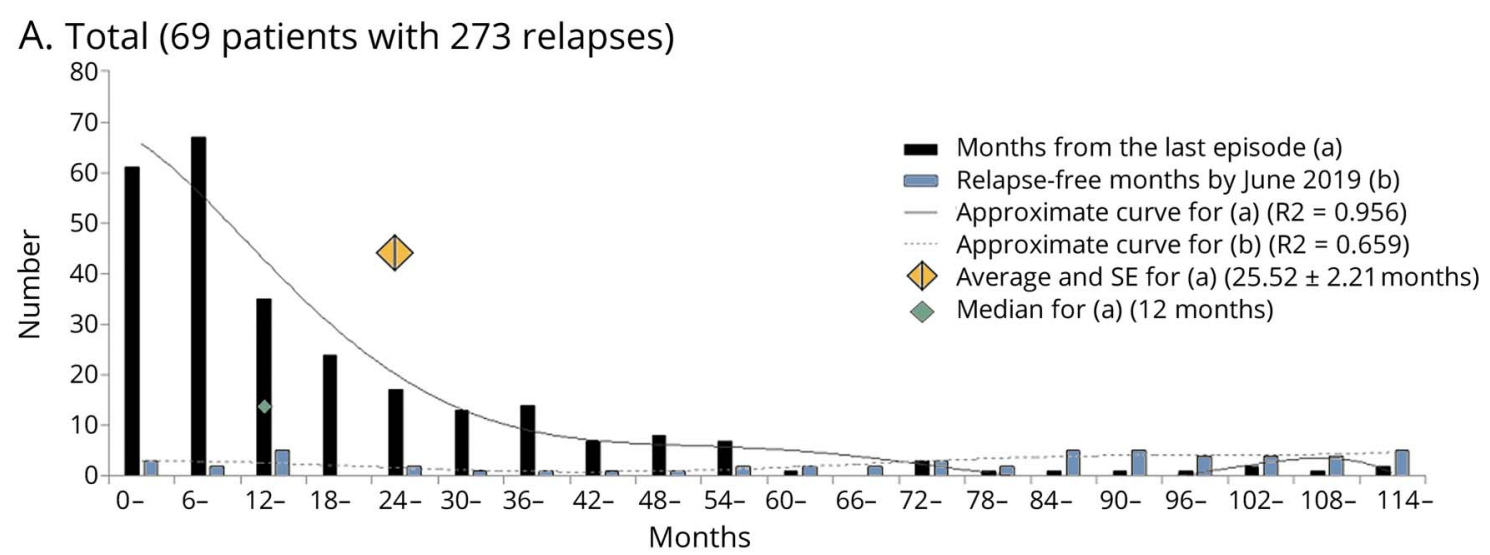

B. Under no treatment (36 patients with 56 relapses)

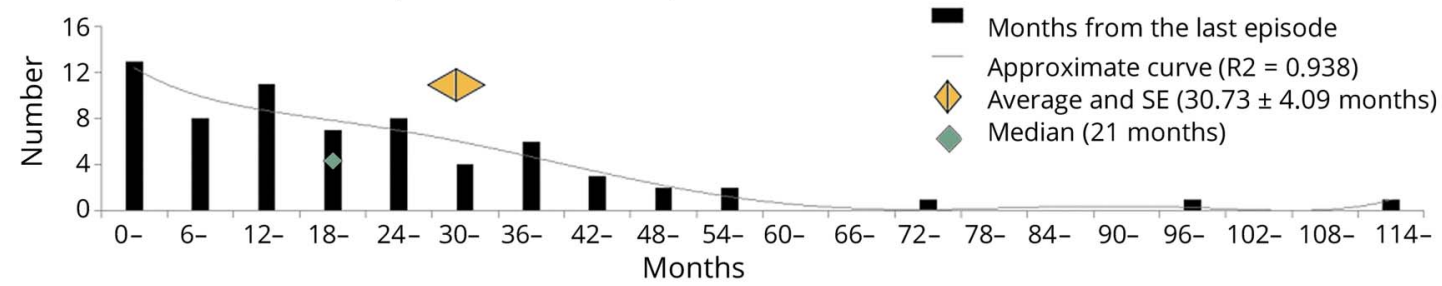

C. Under relapse-preventative therapy (69 patients with 217relapses)

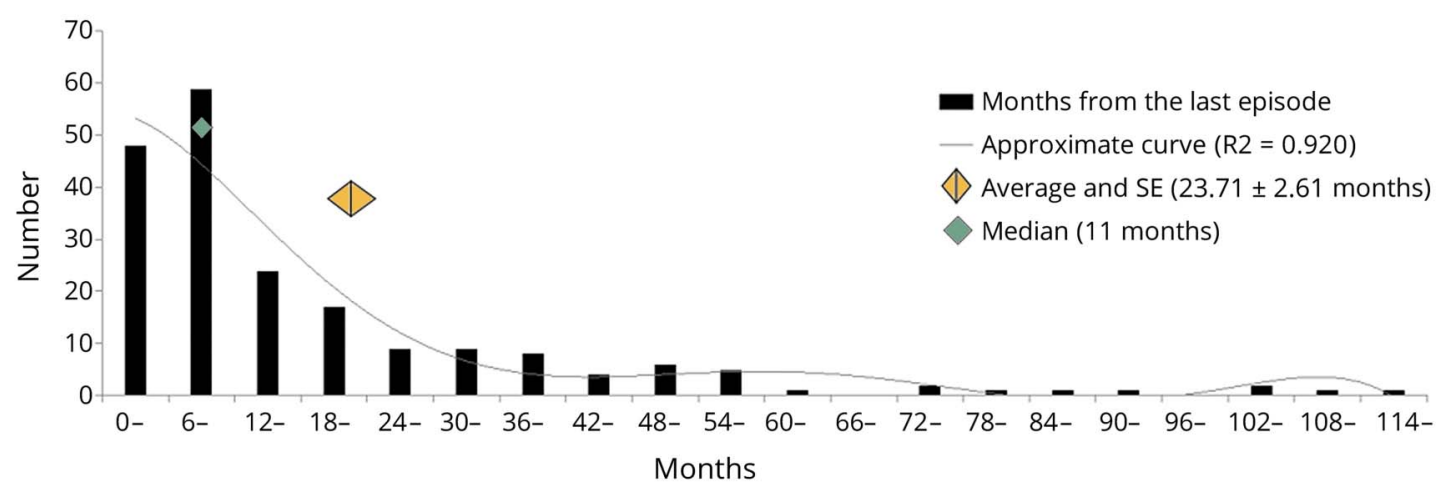

(A) Histograms of the months between 2 tandem attacks (black bars) and the months of relapse-free period by June 2019 (white bars) in all 69 enrolled patients. The center of the gray-colored diamond is the average and its width is the standard error (SE). (B) Histogram of the months between 2 tandem attacks during the medication-free period. (C) Histogram of the months between 2 tandem attacks during the treatment period with oral relapse-preventive therapy. NMOSD = neuromyelitis optica spectrum disorder.

study) and instead dependent solely on whether the patient is in the "clustered" or "nonclustered" period.

Patients should be aggressively treated with adequate amounts of relapse-preventive therapies during the "clustered" period. ${ }^{9,10}$ Empirically, appropriate relapse-preventive treatments may be effective for shifting patients from the "clustered" to the "nonclustered" period. Meanwhile, whether the aggressive treatments should be continued during the "nonclustered" intermittent period is not conclusive at present. ${ }^{11}$ To elucidate the best therapeutic strategy during the nonclustered intermittent period, further clinical studies will be needed. Nevertheless, a minimal level of relapse-preventive therapy should be continued even during the "nonclustered" intermittent period.
A recent report also evaluated the usefulness of previous attacks for predicting the future clinical course. ${ }^{12}$ Although the report did not conclude on the importance of selecting patients with a recent high disease activity for clinical trials, the data suggested that the relapse rate in the following 2 years may be affected by the number of attacks in the past 2 years. Considered together with the results of this study that the relapse rates are significantly different between during the "clustered" and "nonclustered" intermittent periods, allocating the patients without taking the present disease activity into consideration in clinical trials may cause biases in the achieved results. In some relatively old retrospective studies that assessed the effectiveness of relapse-preventive therapies, such as rituximab, azathioprine, or mycophenolate mofetil, the number of attacks in the past 2 years was not 
Figure 4 Kaplan-Meier survival analysis comparing relapse-free periods without medications between 0 and 24 months from onset and later

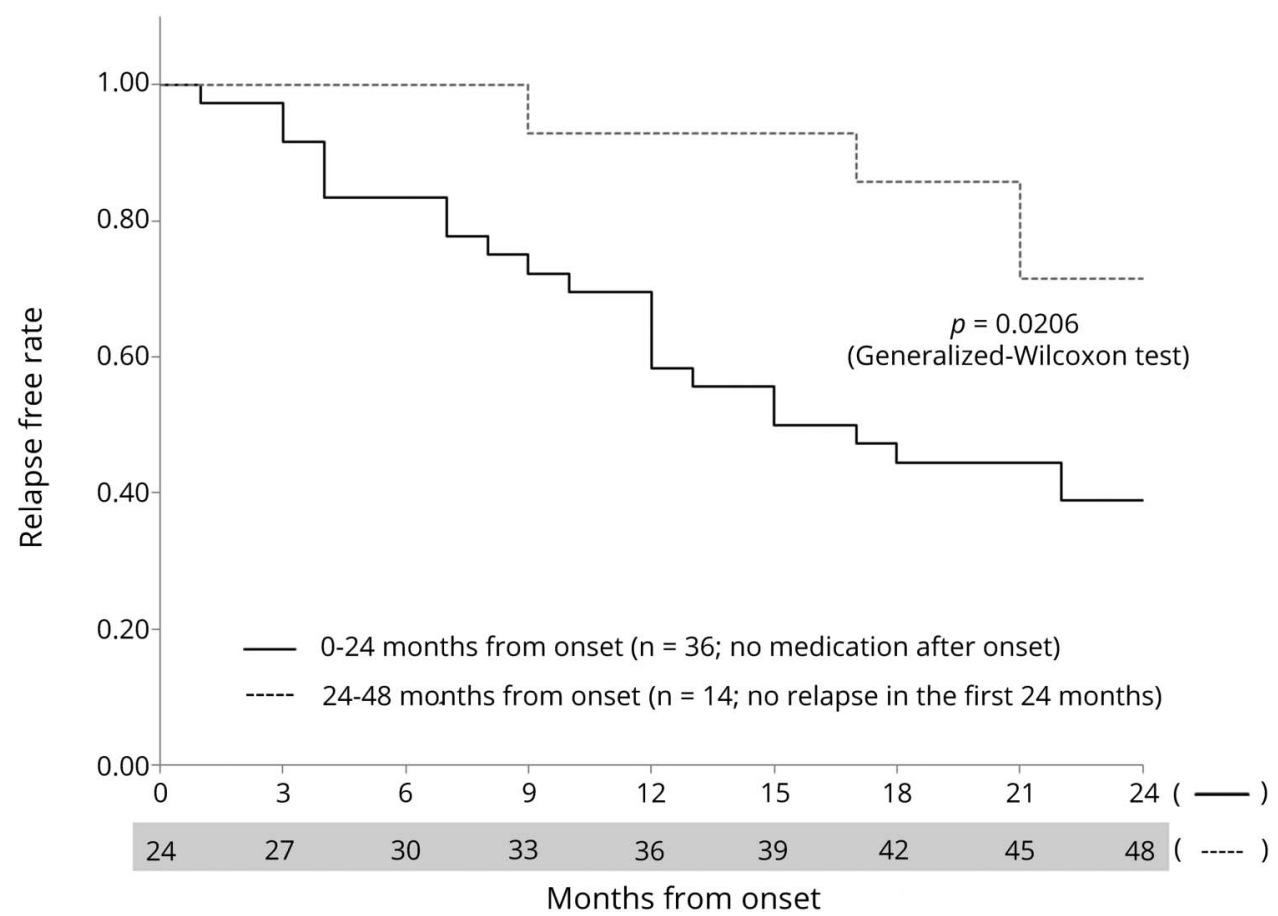

The analysis compared the relapsefree period without relapse-preventive therapies between 0 and 24 months from the onset ( 36 patients with no relapse-preventive therapy) and 24-48 months from the onset (14 patients without relapses in the first 24 months). The latter group represents the "nonclustered" intermittent period status. The former group showed a shorter relapse-free period and a higher relapse rate than the latter group $(p=0.0206$, generalized Wilcoxon test). fully taken into consideration at the participant enrollment stage. ${ }^{13,14}$ As a result, the estimated effectiveness of the studied relapse-preventive therapies may have been weakened because of the inclusion of some patients in the "nonclustered" intermittent period. Meanwhile, intentionally including some patients during their "nonclustered" intermittent period during enrollment for a clinical trial might have some benefits because an important question is whether patients in the "nonclustered" period without a recent history of attacks may benefit similarly from such additional relapse-preventive therapies. Subgroup analysis after dividing the enrolled patients into "clustered" and "nonclustered" groups at enrollment may answer this question.

In the near future, several monoclonal antibody drugs are expected to be released in the market, such as eculizumab, satralizumab, and inebilizumab. ${ }^{15-17}$ These monoclonal antibodies have shown quite a high relapse-preventive efficiency but are very expensive to be continued throughout life. ${ }^{4,18,19}$ The results of this study may indicate that the usage of such expensive monoclonal antibodies could be limited to the "clustered" period and can be spared and switched to lowdose oral corticosteroid or other oral immune suppressants once the patients move into the "nonclustered" intermittent period without relapses for more than 12 months. If such a therapeutic standard is established with evidence, it will surely contribute to reducing the cost of medical expenses.
For reference, in the clinical trials of the abovementioned 3 monoclonal antibodies (PREVENT study for eculizumab, SAkuraSky study for satralizumab, and N-MOmentum study for inebilizumab), the number of clinical attacks in the past 2 years before the enrollment has been fully taken into consideration in all of them, minimizing the risk of biases based on whether participants were in the "clustered" or "nonclustered" period during these clinical trials. ${ }^{15,20}$

Based on this study, the cutoff period for relapse-free status in such clinical studies would be somewhere between 12 months and 24 months. Because the peak relapse-free period from the last clinical attack was $<12$ months, as shown in figure 3 , a 12 month relapse-free status may be better than 12 months per se to consider switching the aggressiveness of relapse-preventive therapies. Meanwhile, approximately $20 \%$ of the patients had their relapses between 12 and 24 months after the last attacks. This fact may imply that a 24-month relapse-free status could be safer than 12 months because that may cover significantly more relapses at up to $70 \%$ of all relapses.

The analyses of this study were based on a cohort that was entirely of Asian ancestry. To determine whether the mixed time course of "clustered" vs "nonclustered" periods observed in this study is generalizable to Caucasian or African American patients with NMOSD, whose clinical severity and disease activity are higher than those of Asian patients, further clinical studies will be helpful. ${ }^{21,22}$ 
Table Sequential pattern of clinical attacks in 50 patients with NMOSD with relapses

\begin{tabular}{llll}
\hline & $\begin{array}{l}\text { Next: } \\
\text { ON }\end{array}$ & $\begin{array}{l}\text { Next: } \\
\text { MY }\end{array}$ & $\boldsymbol{p}$ Value \\
\hline $\begin{array}{l}\text { Previous clinical episode } \\
(\mathbf{n}=\mathbf{2 4 2} \text { sequences) }\end{array}$ & & & \\
\hline ON & 34 & 49 & $p=0.0058\left(\chi^{2}\right)$ \\
\hline MY & 38 & 121 & \\
\hline $\begin{array}{l}\text { Previous 2 clinical episodes } \\
(\mathbf{n}=\mathbf{2 0 2} \text { sequences) }\end{array}$ & & & \\
\hline ON: $\mathbf{2}$ times & 15 & 13 & $\begin{array}{l}p=0.0015 \\
\text { (Cochran- }\end{array}$ \\
\hline ON: $\mathbf{1}$ time, MY: $\mathbf{1}$ time & 21 & 49 & \\
\hline MY: $\mathbf{2}$ times & 21 & 83 & \\
\hline
\end{tabular}

\section{Previous 3 clinical episodes}

( $n=169$ sequences)

\begin{tabular}{lccl}
\hline ON: $\mathbf{3}$ times & 12 & 2 & $\begin{array}{l}p<0.0001 \\
\text { (Cochran- } \\
\text { Armitage) }\end{array}$ \\
\hline ON: $\mathbf{2}$ times, MY: $\mathbf{1}$ time & 11 & 18 & \\
\hline ON: $\mathbf{1}$ time, MY: $\mathbf{2}$ times & 13 & 43 & \\
\hline MY: $\mathbf{3}$ times & 13 & 57 &
\end{tabular}

\begin{tabular}{llll}
\hline $\begin{array}{l}\text { Clustered occurrence period (<6 } \\
\text { months from the last episode) }\end{array}$ & & \\
\hline Previous: ON & 9 & 5 & $\begin{array}{l}p=0.0203 \\
\text { (Fisher exact } \\
\text { test) }\end{array}$
\end{tabular}

\begin{tabular}{llll}
\hline Previous: $\mathbf{M Y}$ & 10 & 30 & \\
\hline $\begin{array}{l}\text { Clustered occurrence period (<12 } \\
\text { months from the last episode) }\end{array}$ & & & \\
\hline Previous: $\mathbf{O N}$ & 18 & 18 & $\begin{array}{l}p=0.0006\left(\chi^{2}\right. \\
\text { test) }\end{array}$ \\
\hline Previous: $\mathbf{M Y}$ & 15 & 65 & \\
\hline
\end{tabular}

\section{Nonclustered period $(\geq 12$}

months from the last episode)

\begin{tabular}{llll}
\hline Previous: $\mathbf{O N}$ & 15 & 34 & $p=0.78\left(\chi^{2}\right.$ test $)$ \\
\hline Previous: $\mathbf{M Y}$ & 27 & 55 &
\end{tabular}

Nonclustered period $(\geq 24$

months from the last episode)

\begin{tabular}{lccl}
\hline Previous: ON & 9 & 20 & $\begin{array}{l}p=1.00 \text { (Fisher } \\
\text { exact test) }\end{array}$ \\
\hline Previous: MY & 17 & 38 & \\
\hline
\end{tabular}

Abbreviations: $\mathrm{MY}=$ myelitis; NMOSD = neuromyelitis optica spectrum disorder; ON = optic neuritis.

Upper = the clinical episode sequence was evaluated in each of the 50 patients with relapses. Clinical episodes with medullary (i.e., hiccups/nausea/vomiting) or cerebral lesions were not counted here. Clinical manifestations of relapses tended to repeat and were significantly influenced by the previous relapse. Lower $=$ sequential patterns of clinical relapses in the clustered occurrence period and in the nonclustered intermittent period were evaluated. Attacks with similar clinical manifestation were likely to sequentially repeat during the clustered occurrence period, but a similar tendency was not observed in the nonclustered intermittent period.
In conclusion, patients with NMOSD showed a mixed clinical course of the "clustered" period with dense relapses and "nonclustered" intermittent period with sparse relapses. Approximately half of the relapses in these patients occurred during the "clustered" period within 12 months of the last clinical attack. Clinicians should pay special attention to whether their patients are presently in the relapse-dense period or intermittent period because this could influence the treatment. Furthermore, the necessity of relapse-preventive therapies during the intermittent period should be established in future clinical studies.

\section{Study funding}

No targeted funding.

\section{Disclosure}

T. Akaishi reports no disclosures. I. Nakashima received speaker honoraria and travel funding from Mitsubishi Tanabe Pharma, Biogen Japan, and Novartis Pharmaceuticals and received research support from LSI Medience Corporation. T. Takahashi, M. Abe, T. Ishii, and M. Aoki report no disclosures. Go to Neurology.org/NN for full disclosures.

\section{Publication history}

Received by Neurology: Neuroimmunology \& Neuroinflammation July 17, 2019. Accepted in final form September 5, 2019.

\section{Appendix Authors}

\begin{tabular}{|c|c|c|c|}
\hline Name & Location & Role & Contribution \\
\hline $\begin{array}{l}\text { Tetsuya } \\
\text { Akaishi, MD, } \\
\text { PhD }\end{array}$ & $\begin{array}{l}\text { Tohoku University, } \\
\text { Sendai, Japan }\end{array}$ & Author & $\begin{array}{l}\text { Designed and } \\
\text { conceptualized the } \\
\text { study, analyzed the } \\
\text { data, and drafted } \\
\text { the manuscript for } \\
\text { intellectual content }\end{array}$ \\
\hline $\begin{array}{l}\text { Ichiro } \\
\text { Nakashima, } \\
\text { MD, PhD }\end{array}$ & $\begin{array}{l}\text { Tohoku Medical } \\
\text { and } \\
\text { Pharmaceutical } \\
\text { University, Sendai, } \\
\text { Japan }\end{array}$ & Author & $\begin{array}{l}\text { Major role in the } \\
\text { acquisition of data and } \\
\text { revised the manuscript } \\
\text { for intellectual content }\end{array}$ \\
\hline $\begin{array}{l}\text { Toshiyuki } \\
\text { Takahashi, } \\
\text { MD, PhD }\end{array}$ & $\begin{array}{l}\text { Tohoku University, } \\
\text { Sendai, Japan }\end{array}$ & Author & $\begin{array}{l}\text { Major role in the } \\
\text { acquisition of data and } \\
\text { revised the manuscript } \\
\text { for intellectual content }\end{array}$ \\
\hline $\begin{array}{l}\text { Michiaki } \\
\text { Abe, MD, } \\
\text { PhD }\end{array}$ & $\begin{array}{l}\text { Tohoku University, } \\
\text { Sendai, Japan }\end{array}$ & Author & $\begin{array}{l}\text { Interpreted the data } \\
\text { and revised the } \\
\text { manuscript for } \\
\text { intellectual content }\end{array}$ \\
\hline $\begin{array}{l}\text { Tadashi } \\
\text { Ishii, MD, } \\
\text { PhD }\end{array}$ & $\begin{array}{l}\text { Tohoku University, } \\
\text { Sendai, Japan }\end{array}$ & Author & $\begin{array}{l}\text { Interpreted the data } \\
\text { and revised the } \\
\text { manuscript for } \\
\text { intellectual content }\end{array}$ \\
\hline $\begin{array}{l}\text { Masashi } \\
\text { Aoki, MD, } \\
\text { PhD }\end{array}$ & $\begin{array}{l}\text { Tohoku University, } \\
\text { Sendai, Japan }\end{array}$ & Author & $\begin{array}{l}\text { Supervised the } \\
\text { research process and } \\
\text { revised the manuscript } \\
\text { for intellectual } \\
\text { content }\end{array}$ \\
\hline
\end{tabular}




\section{References}

1. Wingerchuk DM, Banwell B, Bennett JL, et al. International consensus diagnostic criteria for neuromyelitis optica spectrum disorders. Neurology 2015;85:177-189.

2. Wingerchuk DM, Lennon VA, Pittock SJ, Lucchinetti CF, Weinshenker BG. Revised diagnostic criteria for neuromyelitis optica. Neurology 2006;66:1485-1489.

3. Pittock SJ, Lucchinetti CF. Neuromyelitis optica and the evolving spectrum of autoimmune aquaporin- 4 channelopathies: a decade later. Ann N Y Acad Sci 2016;1366: 20-39.

4. Trebst C, Jarius S, Berthele A, et al. Update on the diagnosis and treatment of neuromyelitis optica: recommendations of the Neuromyelitis Optica Study Group (NEMOS). J Neurol 2014;261:1-16.

5. Kleiter I, Gold R. Present and future therapies in neuromyelitis optica spectrum disorders. Neurotherapeutics 2016;13:70-83.

6. Takahashi T, Fujihara K, Nakashima I, et al. Establishment of a new sensitive assay for anti-human aquaporin-4 antibody in neuromyelitis optica. Tohoku J Exp Med 2006; 210:307-313.

7. Takahashi T, Fujihara K, Nakashima I, et al. Anti-aquaporin-4 antibody is involved in the pathogenesis of NMO: a study on antibody titre. Brain 2007;130:1235-1243.

8. Sato DK, Nakashima I, Takahashi T, et al. Aquaporin-4 antibody-positive cases beyond current diagnostic criteria for NMO spectrum disorders. Neurology 2013;80: 2210-2216.

9. Sherman E, Han MH. Acute and chronic management of neuromyelitis optica spectrum disorder. Curr Treat Options Neurol 2015;17:48.

10. Kessler RA, Mealy MA, Levy M. Treatment of neuromyelitis optica spectrum disorder: acute, preventive, and symptomatic. Curr Treat Options Neurol 2016;18:2.

11. Kimbrough DJ, Fujihara K, Jacob A, et al. Treatment of neuromyelitis optica: Review and recommendations. Mult Scler Relat Disord 2012;1:180-187.
12. Palace J, Lin DY, Zeng D, et al. Outcome prediction models in AQP4-IgG positive neuromyelitis optica spectrum disorders. Brain 2019;142:1310-1323.

13. Mealy MA, Wingerchuk DM, Palace J, Greenberg BM, Levy M. Comparison of relapse and treatment failure rates among patients with neuromyelitis optica: multicenter study of treatment efficacy. JAMA Neurol 2014;71:324-330.

14. Jacob A, Matiello M, Weinshenker BG, et al. Treatment of neuromyelitis optica with mycophenolate mofetil: retrospective analysis of 24 patients. Arch Neurol 2009;66: $1128-1133$.

15. Pittock SJ, Berthele A, Fujihara K, et al. Eculizumab in aquaporin-4-positive neuromyelitis optica spectrum disorder. N Engl J Med 2019;381:614-625.

16. Borisow N, Mori M, Kuwabara S, Scheel M, Paul F. Diagnosis and treatment of NMO spectrum disorder and MOG-encephalomyelitis. Front Neurol 2018;9:888.

17. Agius MA, Klodowska-Duda G, Maciejowski M, et al. Safety and tolerability of inebilizumab (MEDI-551), an anti-CD19 monoclonal antibody, in patients with relapsing forms of multiple sclerosis: results from a phase 1 randomised, placebo-controlled, escalating intravenous and subcutaneous dose study. Mult Scler 2019;25:235-245.

18. Akaishi $\mathrm{T}$, Nakashima I. Efficiency of antibody therapy in demyelinating diseases. Int Immunol 2017;29:327-335.

19. Lin J, Xue B, Li X, Xia J. Monoclonal antibody therapy for neuromyelitis optica spectrum disorder: current and future. Int J Neurosci 2017;127:735-744.

20. Cree BA, Bennett JL, Sheehan M, et al. Placebo-controlled study in neuromyelitis optica-Ethical and design considerations. Mult Scler 2016;22:862-872.

21. Mealy MA, Kessler RA, Rimler Z, et al. Mortality in neuromyelitis optica is strongly associated with African ancestry. Neurol Neuroimmunol Neuroinflamm 2018;5:e468. doi: 10.1212/NXI.0000000000000468.

22. Kim SH, Mealy MA, Levy M, et al. Racial differences in neuromyelitis optica spectrum disorder. Neurology 2018;91:e2089-e2099. 


\section{Neurology \\ Neuroimmunology \& Neuroinflammation}

Neuromyelitis optica spectrum disorders with unevenly clustered attack occurrence Tetsuya Akaishi, Ichiro Nakashima, Toshiyuki Takahashi, et al.

Neurol Neuroimmunol Neuroinflamm 2020;7;

DOI 10.1212/NXI.0000000000000640

This information is current as of November 22, 2019

\section{Updated Information \& Services}

References

Permissions \& Licensing

Reprints including high resolution figures, can be found at: http://nn.neurology.org/content/7/1/e640.full.html

This article cites 22 articles, 1 of which you can access for free at: http://nn.neurology.org/content/7/1/e640.full.html\#\#ref-list-1

Information about reproducing this article in parts (figures,tables) or in its entirety can be found online at:

http://nn.neurology.org/misc/about.xhtml\#permissions

Information about ordering reprints can be found online: http://nn.neurology.org/misc/addir.xhtml\#reprintsus

Neurol Neuroimmunol Neuroinflamm is an official journal of the American Academy of Neurology.

Published since April 2014, it is an open-access, online-only, continuous publication journal. Copyright

Copyright (C) 2019 The Author(s). Published by Wolters Kluwer Health, Inc. on behalf of the American Academy of Neurology.. All rights reserved. Online ISSN: 2332-7812.

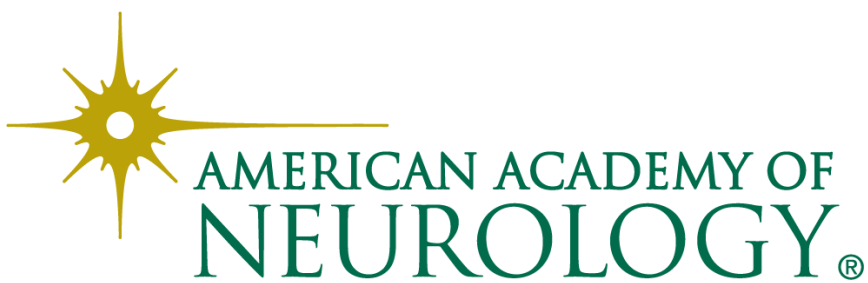

